\title{
Continuous beam steering via controlling light with light on a dielectric metasurface
}

\author{
Fei He ${ }^{1}$, Kevin F MacDonald ${ }^{2}$, Xu Fang ${ }^{*}$ \\ 1. School of Electronics and Computer Science, University of Southampton, Southampton SO17 1BJ, UK \\ 2. Optoelectronics Research Centre and Centre for Photonic Metamaterials, University of Southampton, Southampton SO17 1BJ, UK \\ *x.fang@soton.ac.uk
}

We demonstrate using two coherent light beams to dynamically control the output angle of light from a silicon metasurface. Continuous tuning of $\sim 10^{\circ}$ is observed, suggesting potential applications in Lidar and facial recognition.

Coherent illumination has emerged as a highly versatile and powerful tool for controlling light with light and modulating the physical properties of metamaterials (for recent reviews see [1,2]). The technique is generally considered to be suitable for binary switching, e.g. switching on and off a high-order diffraction beam emitted by a metallic metamaterial. By comparison, there has been little exploration of using the technique for continuous, dynamic modulation. Here we demonstrate using coherent illumination to continuously steer a light beam from an all-dielectric metasurface.

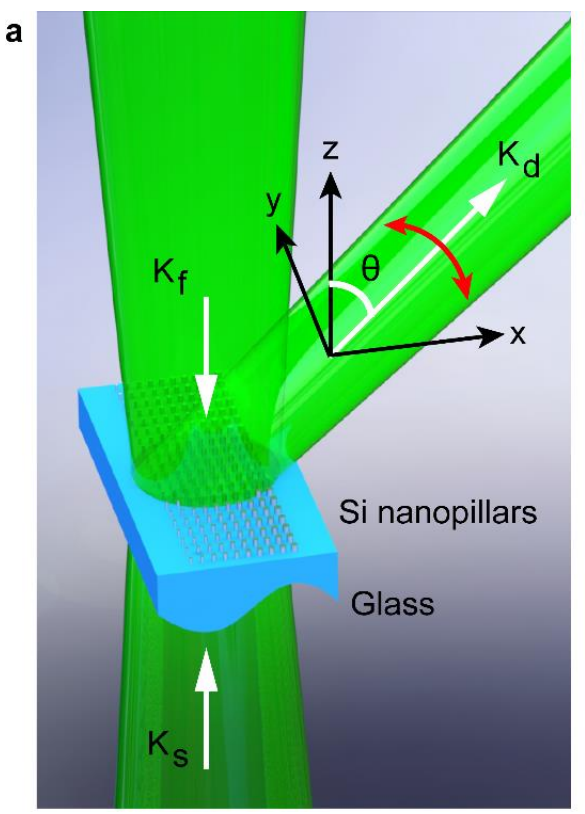

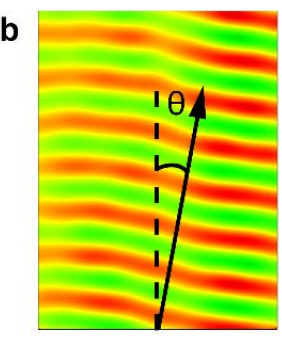

$\theta=10.4^{\circ}$

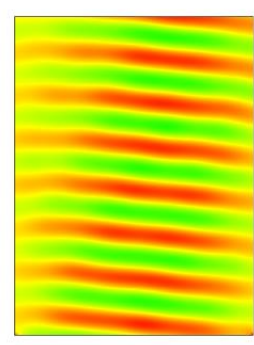

$\theta=5.8^{\circ}$

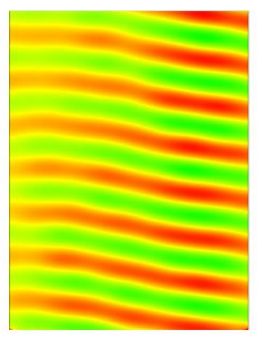

$\theta=9.7^{\circ}$

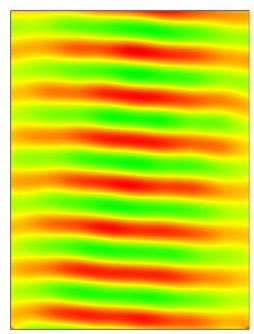

$\theta=2.9^{\circ}$

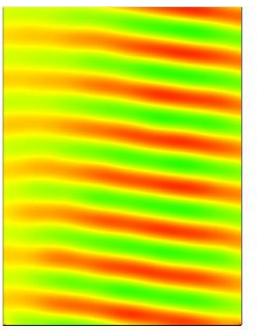

$\theta=7.9^{\circ}$

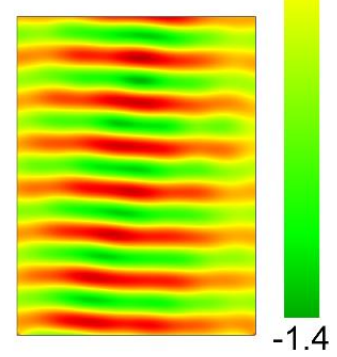

$\theta=1.4^{\circ}$

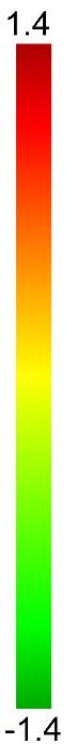

Fig. 1 Coherent illumination of a dielectric metasurface for continuous beam steering. (a) Two counterpropagating light beams $\left(\mathrm{K}_{\mathrm{f}}\right.$ and $\left.\mathrm{K}_{\mathrm{s}}\right)$ illuminate the metasurface at normal incident. The propagation angle $\theta$ of the output beam $\left(\mathrm{K}_{\mathrm{d}}\right)$ deflected into free space can be continuously steered via adjusting the relative phase and intensity of the two incident beams. (b) Numerically simulated electric field distributions of the output beam at different illumination conditions. The amplitude is normalised against that of the free-space input

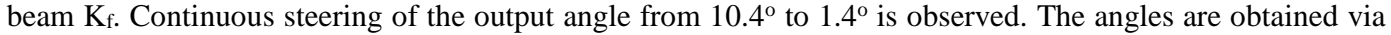
calculating the far-field radiation patterns (not shown here).

Figure 1a schematically illustrates the configuration and operation of the beam-steering metasurface. The metasurface is an array of silicon nanopillars with varied diameters on top of a thick glass substrate. A key aspect of this work is the discovery that, coherent illumination can make the phase delay in light scattering from these pillars tuneable. The output light wave, which is the coherent interference of the light emitted by all these pillars, consequently shows a phase gradient that is also tuneable. By adjusting the relative phase and intensity of the two incident beams, the output angle is tuned from $10.4^{\circ}$ to $1.4^{\circ}$ with the wavefront remains relatively undistorted (Figure 1b).

\section{References}

[1] E. Plum, K. F. MacDonald, X. Fang, D. Faccio, and N. I. Zheludev, "Controlling the Optical Response of 2D Matter in Standing Waves," ACS Photonics 4, 3000 (2017).

[2] X. Fang, K. F. MacDonald, and N. I. Zheludev in, Quantum Photonics: Pioneering Advances and Emerging Applications, R. Boyd, S. Lukishova, and V. Zadkov ed. (Springer, Berlin, 2019). 